\title{
Bell Polynomials and Nonlinear Inverse Relations
}

\author{
Wenchang Chu \\ School of Mathematics and Statistics \\ Zhoukou Normal University \\ Zhoukou (Henan), China \\ Department of Mathematics and Physics \\ University of Salento \\ Lecce, Italy \\ chu. wenchang@unisalento.it
}

Submitted: Apr 22, 2021; Accepted: Nov 4, 2021; Published: Nov 19, 2021

(C) The author. Released under the CC BY-ND license (International 4.0).

\begin{abstract}
By means of the Lagrange expansion formula, we establish a general pair of nonlinear inverse series relations, which are expressed via partial Bell polynomials with the connection coefficients involve an arbitrary formal power series. As applications, two examples are presented with one of them recovering the difficult theorems discovered recently by Birmajer, Gil and Weiner (2012 and 2019).
\end{abstract}

Mathematics Subject Classifications: Primary 05A15, Secondary 05A19

\section{Introduction and Motivation}

For the formal power series $\mathrm{X}(z)$ with its coefficients in a commutative ring

$$
\mathrm{X}(z):=\sum_{k \geqslant 1} x_{k} z^{k} \rightleftharpoons x_{k}:=\left[z^{k}\right] \mathrm{X}(z)
$$

the ordinary partial Bell polynomials (cf. Comtet $[11, \S 3.3]$ ) are defined by

$$
\begin{aligned}
\mathcal{B}_{n, m}(\tilde{x}): & =\mathcal{B}_{n, m}\left(x_{1}, x_{2}, \ldots, x_{n-m+1}\right)=\left[z^{n}\right] \mathrm{X}^{m}(z) \\
& =\sum_{\sigma_{m}(n)}\left(\begin{array}{c}
m \\
m_{1}, m_{2}, \ldots, m_{n}
\end{array}\right) \prod_{k=1}^{n} x_{k}^{m_{k}},
\end{aligned}
$$

where $\left(\begin{array}{c}m \\ m_{1}, m_{2}, \ldots, m_{n}\end{array}\right)$ denotes the usual multinomial coefficient and the sum runs over the subset of partitions of $n$ with $m$-parts represented by $\left(m_{1}, m_{2}, \ldots, m_{n}\right) \in \mathbb{N}_{0}^{n}$ subject to $m_{1}+m_{2}+\cdots+m_{n}=m$ and $m_{1}+2 m_{2}+\cdots+n m_{n}=n$. 
There exist a number of topics related to Bell polynomials. The interested reader can find strange identities in Chu [4, 9, 10], Cvijovic [12] and Hsu [16], summation formulae of special functions in $\mathrm{Hsu}-\mathrm{Chu}$ [17] and $\mathrm{Hsu}-$ Shiue [18], as well as determinant evaluation of Wronskian matrices (cf. Chu [6]).

Let $f(z)$ and $g(z)$ be the two formal power series which are compositional inverses of each other $f \diamond g(z)=g \diamond f(z)=z$. Chou-Hsu-Shiue [3] found the following nonlinear inverse series relations

$$
y_{n}=\sum_{k=1}^{n} \frac{f^{(k)}(a)}{k !} \mathcal{B}_{n, k}(\tilde{x}) \Longleftrightarrow x_{n}=\sum_{k=1}^{n} \frac{g^{(k)}(f(a))}{k !} \mathcal{B}_{n, k}(\tilde{y}) .
$$

Mihoubi [19] derived more examples for polynomial sequences of binomial type.

When the connection coefficients are dependent upon also $n$, Comtet [11, Theorem F, Page 151] recorded a pair of Bell-inverse series relations, which can be reproduced under the replacements $x_{k} \rightarrow-x_{k}$ in the symmetric form:

$$
\left\{\begin{array}{l}
y_{n}=\sum_{k=1}^{n}(-1)^{k}\left(\begin{array}{c}
a n+k \\
k
\end{array}\right) \frac{\mathcal{B}_{n, k}(\tilde{x})}{a n+1}, \\
x_{n}=\sum_{k=1}^{n}(-1)^{k}\left(\begin{array}{c}
a n+k \\
k
\end{array}\right) \frac{\mathcal{B}_{n, k}(\tilde{y})}{a n+1} .
\end{array}\right.
$$

The following Bell inverse series relations are more difficult, which were discovered by Birmajer-Gil-Weiner [1] (see Wang [21] for a different proof via the Lagrange expansion formula):

$$
\left\{\begin{array}{l}
y_{n}=\sum_{k=1}^{n}\left(\begin{array}{c}
1+a n+b k \\
k
\end{array}\right) \frac{\mathcal{B}_{n, k}(\tilde{x})}{1+a n+b k}, \\
x_{n}=\sum_{k=1}^{n}\left(\begin{array}{c}
1-a n-b \\
k
\end{array}\right) \frac{(a n+b k) \mathcal{B}_{n, k}(\tilde{y})}{(a n+b)(1-a n-b)} .
\end{array}\right.
$$

This pair of Bell inversions are recently extended by the same authors [2] with four free parameters $\{a, b, c, d\}$ :

$$
\left\{\begin{array}{l}
y_{n}=\sum_{k=1}^{n}(-1)^{k} \frac{\mathcal{B}_{n, k}(\tilde{x})}{k !} \prod_{i=1}^{k-1}(a n+b k+c i+d), \\
x_{n}=\sum_{k=1}^{n}(-1)^{k} \frac{\mathcal{B}_{n, k}(\tilde{y})}{k !} \frac{\Lambda_{n, k}(b+c)-\Lambda_{n, k}(b)}{c}
\end{array}\right.
$$

where

$$
\Lambda_{n, k}(\rho):=\rho \prod_{j=1}^{k-1}(a n+d j+\rho)
$$


They found also an interesting convolution formula

$$
\sum_{k=1}^{n} \frac{\mathcal{B}_{n, k}(\tilde{y})}{k !} \prod_{i=1}^{k-1}(\lambda-d i)=\sum_{k=1}^{n} \frac{\mathcal{B}_{n, k}(\tilde{x})}{k !} \prod_{j=1}^{k-1}(a n+b k+c j+\lambda) .
$$

The aim of this paper is to generalize further these results by expressing the connection coefficients in terms of an arbitrary formal power series. The main theorems and proofs will be presented in the next section. Then the paper will end with two examples as applications in Section 3. The first example involves the binomial coefficients of HagenRothe type, which recovers the above displayed formulae of Birmajer-Gil-Weiner [1, 2]. Another example contains nonlinear inverse series relations with the Abel coefficients, Cayley numbers (of trees) and harmonic numbers.

\section{Main Results and Proofs}

In combinatorial analysis and enumerative combinatorics, the Lagrange expansion formula (see Comtet [11, §3.8], Gessel [13] and [5, 8]) is fundamental.

Lemma 1. For a formal power series $\varphi(x)$ subject to $\varphi(0) \neq 0$, the functional equation $x=y / \varphi(y)$ determines $y$ as an implicit function of $x$. Then for another formal power series $F(y)$ in the variable $y$, the following expansions hold for both composite series:

$$
\begin{aligned}
& F(y(x))=F(0)+\sum_{n \geqslant 1} \frac{x^{n}}{n}\left[y^{n-1}\right]\left\{F^{\prime}(y) \varphi^{n}(y)\right\}, \\
& \frac{F(y(x))}{1-y \varphi^{\prime}(y) / \varphi(y)}=\sum_{n \geqslant 0} x^{n}\left[y^{n}\right]\left\{F(y) \varphi^{n}(y)\right\} .
\end{aligned}
$$

By repeatedly making use of the above lemma, we shall prove the general theorem.

Theorem 2. Let $\phi(\tau)$ be a formal power series with $\phi(0)=1$ and $\phi^{\prime}(0) \neq 0$. Then we have the generalized nonlinear inverse relations:

$$
\left\{\begin{array}{l}
y_{n}=\sum_{k=1}^{n} U(n, k) \mathcal{B}_{n, k}(\tilde{x}) \\
x_{n}=\sum_{k=1}^{n} V(n, k) \mathcal{B}_{n, k}(\tilde{y})
\end{array}\right.
$$

where the coefficients $U$ and $V$ in the Bell polynomial representations of the sequences are respectively given by

$$
\left\{\begin{array}{l}
U(n, k)=\frac{\lambda}{\lambda+n \alpha+k \beta}\left[\tau^{k}\right] \phi^{\lambda+n \alpha+k \beta}(\tau) \\
V(n, k)=\sum_{i=0}^{n} \frac{\beta-i}{n \alpha+\beta-i}\left(\begin{array}{c}
\frac{i-n \alpha-\beta}{\lambda} \\
k
\end{array}\right) \sum_{j=1}^{n} \frac{(-1)^{i}}{j}\left(\begin{array}{l}
j \\
i
\end{array}\right)\left[\tau^{j-1}\right]\left\{\frac{\tau}{1-\phi(\tau)}\right\}^{j} .
\end{array}\right.
$$


Remark. If we write $\phi(\tau)=1-\tau / \varphi(\tau)$, then $\varphi(\tau)$ will be another formal power series with $\varphi(0) \neq 0$. In this case, the connection coefficients in Theorem 2 can be expressed as

$$
\left\{\begin{array}{l}
U(n, k)=\frac{\lambda}{\lambda+n \alpha+k \beta}\left[\tau^{k}\right]\{1-\tau / \varphi(\tau)\}^{\lambda+n \alpha+k \beta} \\
V(n, k)=\sum_{i=0}^{n} \frac{\beta-i}{n \alpha+\beta-i}\left(\begin{array}{c}
\frac{i-n \alpha-\beta}{\lambda} \\
k
\end{array}\right) \sum_{j=1}^{n} \frac{(-1)^{i}}{j}\left(\begin{array}{c}
j \\
i
\end{array}\right)\left[\tau^{j-1}\right] \varphi^{j}(\tau) .
\end{array}\right.
$$

Proof. For the formal power series $\phi(z)$ and another one $\mathrm{X}(z)=\sum_{k \geqslant 1} x_{k} z^{k}$, as well as an implicit function $\tau$ determined by $\mathrm{X}:=\tau / \phi^{\beta}(\tau)$, define the composite series by

$$
f(z):=z / \phi\left(\tau\left(\mathrm{X}\left(z^{\alpha}\right)\right)\right)
$$

Then there exists the compositional inverse $g$ such that $f \diamond g(z)=g \diamond f(z)=z$ and the power series expansion

$$
g^{\lambda}(z):=\sum_{m \geqslant 0} y_{m} z^{\lambda+m \alpha}
$$

In view of Lagrange inversion formula (4), we can express the coefficient $y_{n}$ as

$$
y_{n}=\left[z^{\lambda+n \alpha}\right] g^{\lambda}(z)=\frac{\lambda}{\lambda+n \alpha}\left[z^{n \alpha}\right] \phi^{\lambda+n \alpha}\left(\tau\left(\mathrm{X}\left(z^{\alpha}\right)\right)\right) .
$$

Applying the expansion formula (4) again, we have also

$$
\begin{aligned}
\phi^{\lambda+n \alpha}(\tau) & =1+\sum_{k \geqslant 1} \frac{X^{k}}{k}(\lambda+n \alpha)\left[\tau^{k-1}\right] \phi^{\prime}(\tau) \phi^{\lambda-1+n \alpha+k \beta}(\tau) \\
& =1+\sum_{k \geqslant 1} \frac{\lambda+n \alpha}{\lambda+n \alpha+k \beta} \mathrm{X}^{k}\left[\tau^{k}\right] \phi^{\lambda+n \alpha+k \beta}(\tau) .
\end{aligned}
$$

Therefore, we get the explicit expression of $y_{n}$ in terms of the Bell polynomials:

$$
y_{n}=\sum_{k \geqslant 1} \frac{\lambda\left[\tau^{k}\right] \phi^{\lambda+n \alpha+k \beta}(\tau)}{\lambda+n \alpha+k \beta}\left[z^{n \alpha}\right] \mathrm{X}^{k}\left(z^{\alpha}\right)=\sum_{k=1}^{n} \frac{\lambda\left[\tau^{k}\right] \phi^{\lambda+n \alpha+k \beta}(\tau)}{\lambda+n \alpha+k \beta} \mathcal{B}_{n, k}(\tilde{x}) .
$$

In order to determine $x_{n}$ in terms of $\left\{y_{k}\right\}$, we examine the coefficient

$$
x_{n}=\left[z^{n \alpha}\right] \mathrm{X}\left(z^{\alpha}\right)=\left[z^{n \alpha}\right] \frac{\tau}{\phi^{\beta}(\tau)} .
$$

Recall that $\phi(z)$ is a formal power series subject to $\phi(0)=1$ and $\phi^{\prime}(0) \neq 0$. We can define another formal power series $\psi(\tau)$ with $\psi(0)=1$ by

$$
\psi(\tau)=\frac{\tau \phi^{\prime}(0)}{\phi(\tau)-1} \rightleftharpoons \frac{\phi(\tau)-1}{\phi^{\prime}(0)}=\tau / \psi(\tau) .
$$


This gives rise to the following expansion

$$
\tau=\sum_{j \geqslant 1}\left\{\frac{\tau}{\psi(\tau)}\right\}^{j} \frac{\left[\eta^{j-1}\right]}{j} \psi^{j}(\eta)=\sum_{j \geqslant 1}\{1-\phi(\tau)\}^{j} \frac{\left[\eta^{j-1}\right]}{j}\left\{\frac{\eta}{1-\phi(\eta)}\right\}^{j} .
$$

Further, $\tau$ is also an implicit formal power series of $z^{\alpha}$ with the constant term equal to zero because of the functional equation $\mathrm{X}\left(z^{\alpha}\right)=\tau / \phi^{\beta}(\tau)$. Hence we can proceed with extracting the coefficient

$$
\begin{aligned}
x_{n} & =\left[z^{n \alpha}\right] \frac{\tau}{\phi^{\beta}(\tau)}=\sum_{j=1}^{n} \frac{\left[\eta^{j-1}\right]}{j} \psi^{j}(\eta)\left[z^{n \alpha}\right]\left\{\frac{\tau^{j}}{\phi^{\beta}(\tau) \psi^{j}(\tau)}\right\} \\
& =\sum_{j=1}^{n} \frac{\left[\eta^{j-1}\right]}{j}\left\{\frac{\eta}{1-\phi(\eta)}\right\}^{j} \sum_{i=0}^{j}(-1)^{i}\left(\begin{array}{l}
j \\
i
\end{array}\right)\left[z^{n \alpha}\right] \phi^{i-\beta}(\tau) \\
& =\sum_{i=0}^{n} \sum_{j=1}^{n}(-1)^{i}\left(\begin{array}{c}
j \\
i
\end{array}\right) \frac{\left[\eta^{j-1}\right]}{j}\left\{\frac{\eta}{1-\phi(\eta)}\right\}^{j}\left[z^{n \alpha+\beta-i}\right] f^{\beta-i}(z) .
\end{aligned}
$$

Keeping in mind that $f$ is the compositional inverse of $g$, we have, in turn, the following expression

$$
\begin{aligned}
{\left[z^{n \alpha+\beta-i}\right] f^{\beta-i}(z) } & =\frac{\beta-i}{n \alpha+\beta-i}\left[z^{n \alpha}\right]\left\{\frac{g(z)}{z}\right\}^{i-n \alpha-\beta} \\
& =\frac{\beta-i}{n \alpha+\beta-i}\left[z^{n \alpha}\right]\left\{1+\left(\frac{g(z)}{z}\right)^{\lambda}-1\right\}^{\frac{i-n \alpha-\beta}{\lambda}} \\
& =\frac{\beta-i}{n \alpha+\beta-i}\left[z^{n \alpha}\right] \sum_{k=1}^{n}\left(\begin{array}{c}
\frac{i-n \alpha-\beta}{\lambda} \\
k
\end{array}\right)\left\{\left(\frac{g(z)}{z}\right)^{\lambda}-1\right\}^{k} \\
& =\frac{\beta-i}{n \alpha+\beta-i} \sum_{k=1}^{n}\left(\begin{array}{c}
\frac{i-n \alpha-\beta}{\lambda} \\
k
\end{array}\right) \mathcal{B}_{n, k}(\tilde{y}) .
\end{aligned}
$$

Substituting this into the last expression of $x_{n}$ and then reorganize the terms, we get the dual relation displayed in (6).

Observe that in Theorem 2, the sequence $\left\{y_{n}\right\}$ is dependent on the parameter " $\lambda$ ". In order to highlight this fact, we introduce the symbol $y_{n}^{\langle\lambda\rangle}$ and the corresponding generating function $\mathrm{Y}^{\langle\lambda\rangle}(z)$ by

$$
\begin{aligned}
& y_{n}^{\langle\lambda\rangle}:=\sum_{k=1}^{n} \frac{\lambda \mathcal{B}_{n, k}(\tilde{x})}{\lambda+n \alpha+k \beta}\left[\tau^{k}\right] \phi^{\lambda+n \alpha+k \beta}(\tau), \\
& \mathrm{Y}^{\langle\lambda\rangle}(z):=\sum_{n=0}^{\infty} z^{\lambda+n \alpha} y_{n}^{\langle\lambda\rangle} .
\end{aligned}
$$

When $\lambda=1$, the superscript " $\langle 1\rangle$ " will be suppressed from those notations. 
Let $\lambda$ and $\mu$ be two natural numbers. Even though there exist no explicit expressions for $\mathrm{Y}^{\langle\lambda\rangle}(z)$, we do have, according to (9), the exponential relation and the convolution formula

$$
\mathrm{Y}^{\langle\lambda\rangle}(z)=\mathrm{Y}^{\lambda}(z) \Longleftrightarrow y_{n}^{\langle\lambda\rangle}=\sum_{k=1}^{n}\left(\begin{array}{l}
\lambda \\
k
\end{array}\right) \mathcal{B}_{n, k}(\tilde{y}) .
$$

Consequently, the exponential laws assert further that

$$
\mathrm{Y}^{\langle\lambda \mu\rangle}(z)=\left\{\mathrm{Y}^{\langle\mu\rangle}(z)\right\}^{\lambda} \text { and } \mathrm{Y}^{\langle\lambda+\mu\rangle}(z)=\mathrm{Y}^{\langle\lambda\rangle}(z) \times \mathrm{Y}^{\langle\mu\rangle}(z)
$$

They correspond to the binomial and convolutional formulae in the following theorem, where the restriction for $\lambda$ and $\mu$ to be natural numbers is released to be complex parameters since (12) is valid for any two complex numbers.

Theorem 3. Let $\lambda$ and $\mu$ be two complex parameters. For the sequence $\left\{y_{n}^{\langle\lambda\rangle}\right\}$ defined by (10), there hold the binomial and convolutional identities

$$
y_{n}^{\langle\lambda \mu\rangle}=\sum_{k=1}^{n}\left(\begin{array}{l}
\lambda \\
k
\end{array}\right) \mathcal{B}_{n, k}\left(\tilde{y}^{\langle\mu\rangle}\right) \quad \text { and } \quad y_{n}^{\langle\lambda+\mu\rangle}=\sum_{k=0}^{n} y_{k}^{\langle\lambda\rangle} y_{n-k}^{\langle\mu\rangle} .
$$

In particular, we have the orthogonal relation

$$
\sum_{k=0}^{n} y_{k}^{\langle\lambda\rangle} y_{n-k}^{\langle-\lambda\rangle}= \begin{cases}1, & n=0 \\ 0, & n>0\end{cases}
$$

which implies the linear inverse series relations

$$
F(n)=\sum_{k=0}^{n} y_{n-k}^{\langle\lambda\rangle} G(k) \Longleftrightarrow G(n)=\sum_{k=0}^{n} y_{n-k}^{\langle-\lambda\rangle} F(k) .
$$

\section{Examples and Applications}

Since the connection coefficients in (7) involve an arbitrary formal power series $\phi(\tau)$, numerous nonlinear inverse series relations and convolution formulae can be derived theoretically from Theorems 2 and 3. We limit to present two significant examples in this section, where the connection coefficients are expressed in terms of Hagen-Rothe coefficients and Abel coefficients, given respectively by

$$
\frac{\lambda}{\lambda+k \beta}\left(\begin{array}{c}
\lambda+k \beta \\
k
\end{array}\right) \text { and } \frac{\lambda}{\lambda+k \beta} \frac{(\lambda+k \beta)^{k}}{k !} .
$$

There have been extensive explorations about these numbers. Generating functions and convolution identities can be found, for example, in Graham-Knuth-Patashnik [15, §5.4], Riordan $[20, \S 4.5]$ and Wilf $[22, \S 2.5]$ as well as in $[7,14]$. 
$\S 3 \mathbf{A}$ Letting $\phi(\tau)=1+\tau$ in $(7)$, we can compute the connection coefficients

$$
\begin{aligned}
U(n, k) & =\frac{\lambda}{\lambda+n \alpha+k \beta}\left[\tau^{k}\right](1+\tau)^{\lambda+n \alpha+k \beta} \\
& =\frac{\lambda}{\lambda+n \alpha+k \beta}\left(\begin{array}{c}
\lambda+n \alpha+k \beta \\
k
\end{array}\right), \\
V(n, k) & =\sum_{i=0}^{n} \frac{\beta-i}{n \alpha+\beta-i}\left(\begin{array}{c}
\frac{i-n \alpha-\beta}{\lambda} \\
k
\end{array}\right)\left(\begin{array}{l}
1 \\
i
\end{array}\right)(-1)^{i+1} \\
& =\frac{\beta-1}{n \alpha+\beta-1}\left(\begin{array}{c}
\frac{1-n \alpha-\beta}{\lambda} \\
k
\end{array}\right)-\frac{\beta}{n \alpha+\beta}\left(\begin{array}{c}
\frac{-n \alpha-\beta}{\lambda} \\
k
\end{array}\right) .
\end{aligned}
$$

According to Theorems 2 and 3, we have the following statements.

- Nonlinear inverse series relations

$$
\left\{\begin{array}{l}
y_{n}^{\langle\lambda\rangle}=\sum_{k=1}^{n}\left(\begin{array}{c}
\lambda+n \alpha+k \beta \\
k
\end{array}\right) \frac{\lambda \mathcal{B}_{n, k}(\tilde{x})}{\lambda+n \alpha+k \beta}, \\
x_{n}=\sum_{k=1}^{n}\left\{\left(\begin{array}{c}
\frac{1-n \alpha-\beta}{\lambda} \\
k
\end{array}\right) \frac{(\beta-1) \mathcal{B}_{n, k}\left(\tilde{y}^{\langle\lambda\rangle}\right)}{n \alpha+\beta-1}-\left(\begin{array}{c}
\frac{-n \alpha-\beta}{\lambda} \\
k
\end{array}\right) \frac{\beta \mathcal{B}_{n, k}\left(\tilde{y}^{\langle\lambda\rangle}\right)}{n \alpha+\beta}\right\} .
\end{array}\right.
$$

When $\lambda=1$, this pair of inversions reduce to (1) due to Birmajer-Gil-Weiner [1]. Furthermore, the above inversions are equivalent to those displayed in (2), discovered also by Birmajer-Gil-Weiner [2]. This can be verified by making substitutions $\alpha \rightarrow-a / c, \beta \rightarrow-b / c, \lambda \rightarrow-d / c$ and then replacements $x_{k} \rightarrow-c x_{k}$ and $y_{k} \rightarrow d y_{k}$.

- Binomial and convolutional identities

$$
\begin{gathered}
\sum_{k=1}^{n}\left(\begin{array}{l}
\lambda \\
k
\end{array}\right) \mathcal{B}_{n, k}\left(\tilde{y}^{\langle\mu\rangle}\right)=\sum_{k=1}^{n}\left(\begin{array}{c}
\lambda \mu+n \alpha+k \beta \\
k
\end{array}\right) \frac{(\lambda \mu) \mathcal{B}_{n, k}(\tilde{x})}{\lambda \mu+n \alpha+k \beta}, \\
\sum_{k=0}^{n} y_{k}^{\langle\lambda\rangle} y_{n-k}^{\langle\mu\rangle}=\sum_{k=1}^{n}\left(\begin{array}{c}
\lambda+\mu+n \alpha+k \beta \\
k
\end{array}\right) \frac{(\lambda+\mu) \mathcal{B}_{n, k}(\tilde{x})}{\lambda+\mu+n \alpha+k \beta} .
\end{gathered}
$$

Analogously, one can check that the second identity implies a formula due to Birmajer-Gil-Weiner [2, Proposition 5]; while the first one contains, as special cases, (3) and three further identities found by the same authors [2, Lemma 18 and Propositions 19-20].

$\S 3 \mathrm{~B}$ Instead, for $\phi(\tau)=e^{-\tau}$ in Theorem 2, the corresponding connection coefficients can be expressed as:

$$
U(n, k)=\frac{\lambda}{\lambda+n \alpha+k \beta}\left[\tau^{k}\right] e^{-\tau(\lambda+n \alpha+k \beta)}=\frac{\lambda(-1)^{k}}{\lambda+n \alpha+k \beta} \frac{(\lambda+n \alpha+k \beta)^{k}}{k !},
$$




$$
V(n, k)=\sum_{i=0}^{n} \frac{\beta-i}{n \alpha+\beta-i}\left(\begin{array}{c}
\frac{i-n \alpha-\beta}{\lambda} \\
k
\end{array}\right) \sum_{j=1}^{n} \frac{(-1)^{i}}{j}\left(\begin{array}{l}
j \\
i
\end{array}\right)\left[\tau^{j-1}\right]\left\{\frac{\tau}{1-e^{-\tau}}\right\}^{j}
$$

Recall that " $1-e^{-\tau "}$ is the compositional inverse of " $-\ln (1-\tau)$ ". By making use of Lemma 1, we have

$$
\left[\tau^{j-1}\right]\left\{\frac{\tau}{1-e^{-\tau}}\right\}^{j}=-j\left[\tau^{j}\right] \ln (1-\tau)=1 .
$$

Therefore, we can evaluate further

$$
\sum_{j=1}^{n} \frac{(-1)^{i}}{j}\left(\begin{array}{l}
j \\
i
\end{array}\right)\left[\tau^{j-1}\right]\left\{\frac{\tau}{1-e^{-\tau}}\right\}^{j}=\sum_{j=1}^{n} \frac{(-1)^{i}}{j}\left(\begin{array}{c}
j \\
i
\end{array}\right)= \begin{cases}H_{n}, & i=0 \\
\frac{(-1)^{i}}{i}\left(\begin{array}{c}
n \\
i
\end{array}\right), & 1 \leqslant i \leqslant n\end{cases}
$$

where $H_{n}=\sum_{k=1}^{n} \frac{1}{k}$ stands for the $n$th harmonic number. Therefore, we have derived an explicit formula

$$
V(n, k)=\frac{\beta H_{n}}{n \alpha+\beta}\left(\begin{array}{c}
\frac{-n \alpha-\beta}{\lambda} \\
k
\end{array}\right)+\sum_{i=1}^{n}(-1)^{i}\left(\begin{array}{c}
n \\
i
\end{array}\right)\left(\begin{array}{c}
\frac{i-n \alpha-\beta}{\lambda} \\
k
\end{array}\right) \frac{\beta-i}{i(n \alpha+\beta-i)} .
$$

In order to simplify the last sum " $S$ " with respect to $i$, we examine the function

$$
\left(\begin{array}{c}
\frac{x-n \alpha-\beta}{\lambda} \\
k
\end{array}\right) \frac{\beta-x}{x(n \alpha+\beta-x)}=P(x)+\frac{\beta}{x(n \alpha+\beta)}\left(\begin{array}{c}
\frac{-n \alpha-\beta}{\lambda} \\
k
\end{array}\right)
$$

where $P(x)$ is a polynomial of degree $k-1$ in $x$. Denote by $\Delta$ the forward difference operator of unit increment with respect to $x$. Then we can compute, in the closed form, the following finite sum

$$
\begin{aligned}
S(x) & :=\sum_{i=0}^{n}(-1)^{i}\left(\begin{array}{c}
n \\
i
\end{array}\right)\left(\begin{array}{c}
\frac{x+i-n \alpha-\beta}{\lambda} \\
k
\end{array}\right) \frac{\beta-i-x}{(x+i)(n \alpha+\beta-i-x)} \\
& =(-1)^{n} \Delta^{n}\left\{P(x)+\frac{\beta}{x(n \alpha+\beta)}\left(\begin{array}{c}
\frac{-n \alpha-\beta}{\lambda} \\
k
\end{array}\right)\right\} \\
& =\frac{n ! \beta}{(x)_{n+1}(n \alpha+\beta)}\left(\begin{array}{c}
\frac{-n \alpha-\beta}{\lambda} \\
k
\end{array}\right) .
\end{aligned}
$$

Hence we can evaluate " $S$ " by the limiting process below

$$
\begin{aligned}
S & =\lim _{x \rightarrow 0}\left\{S(x)-\left(\begin{array}{c}
\frac{x-n \alpha-\beta}{\lambda} \\
k
\end{array}\right) \frac{\beta-x}{x(n \alpha+\beta-x)}\right\} \\
& =\lim _{x \rightarrow 0} \frac{1}{x}\left\{\frac { n ! \beta } { ( 1 + x ) _ { n } ( n \alpha + \beta ) } \left(\begin{array}{c}
\left.\left.\frac{-n \alpha-\beta}{\lambda}\right)-\left(\begin{array}{c}
\frac{x-n \alpha-\beta}{\lambda} \\
k
\end{array}\right) \frac{\beta-x}{n \alpha+\beta-x}\right\} \\
\end{array}=\frac{1}{n \alpha+\beta}\left(\begin{array}{c}
\frac{-n \alpha-\beta}{\lambda} \\
k
\end{array}\right)\left\{1+\sum_{i=1}^{k-1} \frac{\beta}{n \alpha+\beta+i \lambda}\right\}-\frac{\beta H_{n}}{n \alpha+\beta}\left(\begin{array}{c}
\frac{-n \alpha-\beta}{\lambda} \\
k
\end{array}\right) .\right.\right.
\end{aligned}
$$


This yields consequently the explicit expression for the connection coefficient

$$
V(n, k)=\frac{1}{n \alpha+\beta}\left(\begin{array}{c}
\frac{-n \alpha-\beta}{\lambda} \\
k
\end{array}\right)\left\{1+\sum_{i=1}^{k-1} \frac{\beta}{n \alpha+\beta+i \lambda}\right\} .
$$

According to Theorems 2 and 3, we have reached the the following statements.

- Nonlinear inverse series relations

$$
\left\{\begin{array}{l}
y_{n}^{\langle\lambda\rangle}=\sum_{k=1}^{n}(-1)^{k} \frac{(\lambda+n \alpha+k \beta)^{k}}{k !} \frac{\lambda \mathcal{B}_{n, k}(\tilde{x})}{\lambda+n \alpha+k \beta}, \\
x_{n}=\sum_{k=1}^{n} \frac{\mathcal{B}_{n, k}\left(\tilde{y}^{\langle\lambda\rangle}\right)}{n \alpha+\beta}\left(\begin{array}{c}
\frac{-n \alpha-\beta}{\lambda} \\
k
\end{array}\right)\left\{1+\sum_{i=1}^{k-1} \frac{\beta}{n \alpha+\beta+i \lambda}\right\} .
\end{array}\right.
$$

- Binomial and convolutional identities

$$
\begin{aligned}
& \sum_{k=1}^{n}\left(\begin{array}{l}
\lambda \\
k
\end{array}\right) \mathcal{B}_{n, k}\left(\tilde{y}^{\langle\mu\rangle}\right)=\sum_{k=1}^{n}(-1)^{k} \frac{(\lambda \mu+n \alpha+k \beta)^{k}}{k !} \frac{(\lambda \mu) \mathcal{B}_{n, k}(\tilde{x})}{\lambda \mu+n \alpha+k \beta}, \\
& \sum_{k=0}^{n} y_{k}^{\langle\lambda\rangle} y_{n-k}^{\langle\mu\rangle}=\sum_{k=1}^{n}(-1)^{k} \frac{(\lambda+\mu+n \alpha+k \beta)^{k}}{k !} \frac{(\lambda+\mu) \mathcal{B}_{n, k}(\tilde{x})}{\lambda+\mu+n \alpha+k \beta} .
\end{aligned}
$$

- By specifying $\alpha=0$ and $\beta=\lambda=1$ further in (17), we derive the following remarkable inverse pair, where harmonic numbers and the Cayley numbers (of trees) emerge as connection coefficients.

$$
\left\{\begin{array}{l}
y_{n}=\sum_{k=1}^{n}(-1)^{k} \frac{(k+1)^{k-1}}{k !} \mathcal{B}_{n, k}(\tilde{x}) \\
x_{n}=\sum_{k=1}^{n}(-1)^{k} H_{k} \mathcal{B}_{n, k}(\tilde{y})
\end{array}\right.
$$

\section{Acknowledgement}

The author is sincerely grateful to the anonymous referee for the careful reading and helpful suggestions that contributed significantly to improving the manuscript during the revision.

\section{References}

[1] D. Birmajer, J. B. Gil, and M. D. Weiner, Some convolution identities and an inverse relation involving partial Bell polynomials, Electron J. Combin., 19(4) (2012), \#P34.

[2] D. Birmajer, J. B. Gil, and M. D. Weiner, A family of Bell transformations, Discrete Math., 342 (2019), 38-54. 
[3] W. S. Chou, L. C. Hsu, and Peter J. S. Shiue, Application of Faà di Bruno's formula in characterization of inverse relations, J. Comput. Appl. Math., 190 (2006), 151-169.

[4] W. Chu, A partition identity on binomial coefficients and its applications, Graphs Combin., 5 (1989), 197-200.

[5] W. Chu, Generating functions and combinatorial identities, Glas. Mat., 33 (1998), $1-12$.

[6] W. Chu, The Faà di Bruno formula and determinant identities, Linear Multilinear Algebra, 54:1 (2006), 1-25.

[7] W. Chu, Elementary proofs for convolution identities of Abel and Hagen-Rothe, Electron. J. Combin., 17 (2010), \#N24.

[8] W. Chu, Derivative inverse series relations and Lagrange expansion formula, Int. J. Number Theory, 9(4) (2013), 1001-1013.

[9] W. Chu, Logarithms of a binomial series: Extension of a series of Knuth, Math. Commun., 24(1) (2019), 83-90.

[10] W. Chu, Bell polynomials and formulae for derivatives, Ann. Polon. Math., 122(2) (2019), 143-151.

[11] L. Comtet, Advanced Combinatorics, Dordrecht-Holland, The Netherlands, 1974 (Chap. III).

[12] D. Cvijović, New identities for the partial Bell polynomials, Appl. Math. Lett., 24 (2011), 1544-1547.

[13] I. M. Gessel, Lagrange inversion, J. Combin. Theory Ser. A, 144 (2016), 212-249.

[14] H. W. Gould, Some generalizations of Vandermonde's convolution, Amer. Math. Monthly, 63 (1956), 84-91.

[15] R. L. Graham, D. E. Knuth, and O. Patashnik, Concrete Mathematics, AddisonWesley Publ. Company, Reading, Massachusetts, 1989.

[16] L. C. Hsu, Finding some strange identities via Faà di Bruno's formula, J. Math. Res. Expos., 13(2) (1993), 159-165.

[17] L. C. Hsu and W. Chu, A kind of asymptotic expansion using partitions, Tôhoku Math. J., 43(2) (1991), 235-242.

[18] L. C. Hsu and P. J-S. Shiue, Cycle indicators and special functions, Ann. Comb., 5 (2001), 179-196.

[19] M. Mihoubi, Partial Bell polynomials and inverse relations, J. Integer Seq., 13 (2010), Article 10.4.5.

[20] J. Riordan, Combinatorial Identities, John Wiley \& Sons, New York, 1968.

[21] J. Wang, Nonlinear inverse relations for Bell polynomials via the Lagrange inversion formula, J. Integer Seq., 22 (2019), Article 19.3.8.

[22] H. S. Wilf, Generatingfunctionology (second edition), Academic Press Inc., London, 1994. 